\title{
Micro-satellite Constellation-The Challenges and the Possible Contribution of VNSC
}

\author{
Vu Viet Phuong and Pham Anh Tuan \\ Vietnam National Satellite Center, Vietnam Academy of Science and Technology, Hanoi 10000, Vietnam
}

\begin{abstract}
In response to the fast evolution of micro-satellite, the "AMC (Asian micro-satellite consortium)” is established to promote and accelerate the development of micro-satellite and its applications including future projects such as "a super constellation”. Through this paper, we want to share our views on the challenges facing implementation of micro constellation by many institutions with their benefit requirements and also the possible contribution from Vietnam National Satellite Center to this project.
\end{abstract}

Key words: Micro-satellite, constellation, continuous observation, Asian satellite.

\section{Introduction}

With the rapid advancement of downsizing, the space components including payload caused the development of micro-satellite not only from the advantages for education and new product experiments due to low-cost and quick development, but also from the rapidly increasing capabilities of micro-satellite [1]. There have been more and more research institutions concentrating on studying and developing the micro-satellite today. To accelerate the development of micro-satellite and its utilization and increase the value added products from remote-sensing system with micro-satellite, an "Asian micro-satellite constellation" based on the international consortium is now intended for implementation [2]. Besides the "super benefits" obtained from the constellation which will be discussed in many other papers [3], there are also many challenges during implementation and operations of a shared constellation. With this paper, we will share our views on this matter and discuss the solutions and actions that are considered to run a shared constellation.

Like any shared resource system, there are interests, disquiets, and responsibilities shared by all members

Corresponding author: Vu Viet Phuong, master, research fields: communication, satellite technology. E-mail: vvphuong@vnsc.org.vn. that should be acknowledged, accepted, and/or agreed upon during the initial planning stages and re-studied throughout the whole process. All members must firstly analyze and solve the tradeoff "values added" of sharing constellation for "the loss" due to lacking of full control of their contributed satellites.

The interests and disquiets that should be shared among members are:

- To protect the rights to all members, for example, all members should involve and make decision together regarding the design, development, operations, etc.;

- All members must understand the objectives and together propose the system in compliance with the principles and regulations.

Shared responsibilities related to all members should include:

- to participate actively in the design, development, implementation and operations of the system;

- to fulfill these responsibilities throughout the life of the project;

- to work to achieve consensus with other members in the event of conflicts.

\section{The Challenge Facing Constellation and the Proposed Solutions to Overcome}

In response to the call from constellation creators, 
VNSC (Vietnam National Satellite Center) is studying the possibility of constellation's implementation and wanting to share our points of view in this paper. As the alliance network, this micro-satellites constellation will possess all characteristics of a network sharing. Here are the key considerations in review of the collaboration of a satellite constellation network.

The advantages of a shared constellation are:

- The constellation can provide a full, continuous global coverage;

- It helps to share obtained data in efficient manner if it is under good control;

- It enable resources sharing including costs and risks;

- It also helps promote micro-satellite development (common quality, standards and technology);

- Its flexibility allows rapid growth.

The disadvantages or challenge of a shared constellation are:

- The constellation is normally complicated and long time-consuming management due to dependency (finding the mutual agreement);

- It generates conflict of interests, how to choose?

- It can become dangerous if it is lost control;

- Common technology may be difficult to share;

- The difficulty of securing commitment in the future may lead to rapid decline.

To solve the above matters, we suggest performing a preparatory survey on the project. This survey will study carefully feasibility of the project since early startup project time, to analyze and evaluate all criteria, tools, process modeling, and techniques to perform the system designs, implementation and system operation.

Here may be the list of topics served as key considerations in the feasibility study for this project besides other normal topics as usual.

Firstly, to study the economic and social feasibility, we need:

- to define all stakeholders of project who are member institutions, project team, future customers, government agencies/regulators, social community, the public etc.;

- to assess the influences, benefits, impacts of the project on each stakeholder [5].

Secondly, technical feasibility has the responsibility to assess:

- the mission/payload and constellation design which meet the expectation of all members. Different objectives from members can have different requirements of mission/payload and observation revisited time, etc.;

- other common technical requirements like data architecture standard, communication protocols, modulation... (perhaps using the recommended standards from the Consultative Committee for Space Data Systems [6]); link budget and related ground station requirements including networking in between ground stations; minimum requirements for satellite parameters and configuration; possibility of technical transfer/collaboration, etc..

Thirdly, there are many subjects to be considered in the operation feasibility study. This is a critical aspect that needs to be assessed to guarantee success of the project. At this step, we want to focus on the way to balance/manage the risks from conflicts of interest due to the limitation of shared resources (for example, request the images at the same time by two or more members, while there are limitations of storage, communication window for data downlink...) and come out with the process to develop the mechanism of constellation operation management.

We also need to define the method for monitoring and controlling of the satellite operation and the observation mission planning system; pros and cons of management concentration or distribution (at each member's site). Other operation matters like priority management-conflict of interest in observation planning. User interface and data distribution procedure also need to be considered.

Last but not least, other studies in schedule, legal, international culture, finance (especially operations cost for shared equipment/systems/human resource), 
etc., should also be done together as mentioned in above studies to ensure success of the project.

Members should be encouraged to actively join the project to prove the real world. Two pilot micro-satellites with constellation payload could be developed and launched by two pioneering institutions.

\section{The Possible Contribution from VNSC}

In 2006, "The Strategy for Research and Application of Space Technology towards 2020” [4] was approved by Vietnam's Prime Minister. It plays the role as the master plan for the development of space technology in Vietnam. In the coming year-2008, Vietnam formulated the "national target program to respond to climate change" with the purpose to evaluate the impacts caused by climate change and review its countermeasures. In this program, the "promotion of scientific and technological activities" is listed as one of the most important aspects. Since then, Vietnam has fostered research in space science and technology. In order to achieve the ultimate goal of the master plan in building the facilities and introducing technology for the design, production, and operation of earth observation satellites and subsequently domestically produce satellite by 2020, a specialized space agency needs to be established. On September 16, 2011, VNSC was established by the Prime Minister of Vietnam. VNSC is a national research agency administered by the Vietnam Academy of Science and Technology, responsible for research, technology development, application, and capacity built in satellite technology. VNSC is also a core organization involved in receiving, management and implementation of the Vietnam Space Center Project. Right from early days, VNSC has concentrated on satellite development especially on small satellite technology by building the roadmap with clear milestones until 2020 as showed in Fig. 1.

Our method to approach satellite development is starting to research from simple to complex project with

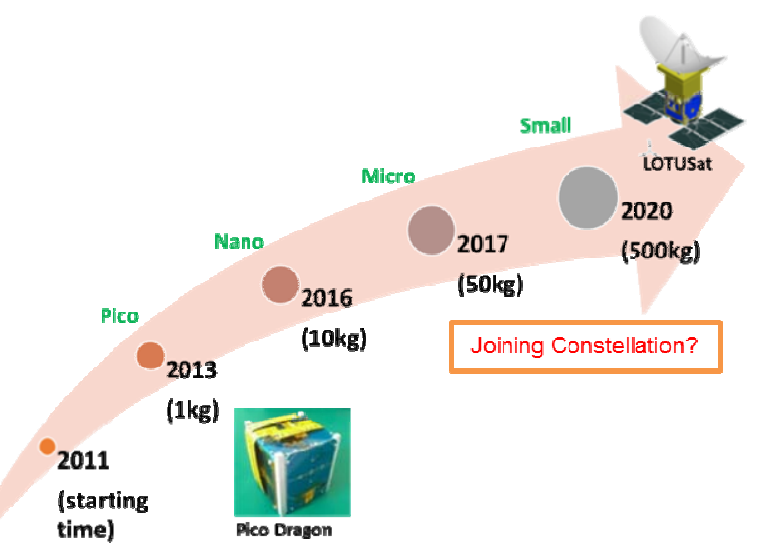

Fig. 1 The milestone schedule of the satellite development at VNSC [7].

the size increased from small to big, finding opportunities for technical assistance from international cooperations... VNSC successfully researched and produced a pico-satellite named PicoDragon under the technical consultancy from Japan between 2011 and 2013. This $1 \mathrm{~kg}$ and $10 \mathrm{~cm} \times$ $10 \mathrm{~cm} \times 11.35 \mathrm{~cm}$ satellite was successfully launched on August 4, 2013 via HTV4 [8]. In addition, we have established other collaborations to gain more experiences and acquire information in order to promote research and applications of space technology since the very beginning of VNSC. At the 15th APRSAF (Asia Pacific Regional Space Agency Forum) in 2008, Vietnam Academy of Science and Technology joined the Sentinel Asia Project and became an official member of APRSAF-Satellite (STAR Project) which later became known as UNIFORM Project. Above all, VNSC is responsible for managing and running the Vietnam Space Center Project, the biggest one ever in the field of science and technology in Vietnam. The main objectives of this project is to improve climate change and disaster countermeasure by launching high resolution Earth observation satellites. It is also important for Vietnam to self-produce and utilize small satellites by 2020 using Vietnamese infrastructure and personnel whose skills are equipped through capacity building and technology transfer programs from overseas. Through this project, a micro-satellite will also be designed, 
manufactured and scheduled for launch in 2017. It could serve as an element of micro-satellite constellation for this project.

\section{Conclusions}

With many advantages, an "Asian micro-satellite constellation" can play the role as an effective continuous earth observation system. It also serves as a good evidence to prove the bright future of micro-satellite in coming years. However, there are still many issues to be discussed and resolved, especially when the constellation will be implemented by many institutions in many countries. To make the dream come true, hard work is expected from founder team in order to collaborate with different entities and remove all obstacles.

\section{References}

[1] Helvanjian, H., and Janson, S. W. 2008. Small Satellites: Past, Present, and Future. Virginia: American Institute of Aeronautics and Astronautics, Inc.
[2] Takahashi, Y. 2013. "Purpose of Asian Micro-satellite Consortium.” Presented at the 34th Asian Conference on Remote Sensing 2013, Bali, Indonesia.

[3] Sandau, R., Nakasuka, S., Kawashima, R., and Sellers, J. 2012. Novel Ideas for Nanosatellite Constellation Missions. Paris: International Academy of Astronautics.

[4] Decision of Vietnam Prime Minister No. 137/2006/QD-TTg. 2006. The Strategy for Research and Application of Space Technology towards 2020. Hanoi: Government of Vietnam.

[5] Reynolds, S. J., Schultz, F. C., and Hekman, D. R. 2006. "Stakeholder Theory and Managerial Decision-Making: Constraints and Implications of Balancing Stakeholder Interests.” Journal of Business Ethics 64 (3): 285-301. Doi: 10.1007/s10551-005-5493-2.

[6] The Consultative Committee for Space Data Systems Recommended Standards (Blue Books). Accessed October 10, 2014. http://public.ccsds.org/publications/BlueBooks.aspx.

[7] Tuan, P. A., and Phuong, V. V. 2013. "Earth Observation Satellite Development in Vietnam.” Presented at the 64th International Astronautical Congress, Beijing, China.

[8] Phuong, V. V. 2014. "A Successful Partnership.” Accessed October 2014. http://global.jaxa.jp/article/interview/2014/vol82/index_e. html. 